\title{
Consumo de ansiolíticos benzodiazepínicos: uma correlação entre dados do SNGPC e indicadores sociodemográficos nas capitais brasileiras
}

\author{
Consumption of anxiolytic benzodiazepines: a correlation between \\ SNGPC data and sociodemographic indicators in Brazilian capitals
}

Ângelo José Pimentel de Azevedo ${ }^{1}$
Aurigena Antunes de Araújo ${ }^{2}$
Maria Ângela Fernandes Ferreira ${ }^{1}$
${ }^{1}$ Pós-Graduação em Saúde Coletiva, Universidade Federal do Rio Grande do Norte (UFRN). Av. Senador Salgado Filho 1787, Lagoa Nova. 59075-000 Natal RN Brasil. angelodeveza@gmail.com ${ }^{2}$ Departamento de Biofísica e Farmacologia, Centro de Biociências, UFRN.

\begin{abstract}
The scope of this article is to determine the distribution and frequency of consumption of anxiolytic benzodiazepines and the correlation between consumption and demographic, epidemiological, economic and social characteristics. It is an ecological study with a sample of 27 state capitals. Data collection was performed through the ANVISA database for the dispensation of Alprazolam, Bromazepam, Clonazepam, Diazepam and Lorazepam in 2010-2012, the 2010 Demographic Census (IBGE), DATASUS and Medical Demographic Research. Descriptive statistical analysis and multiple linear regression analyses were performed for data analysis. The northern region has capitals with the lowest and the southeast has capitals with the highest average consumption of these products. The average consumption for the population of all capitals was 3.60 DHD. Alprazolam is the drug most dispensed by pharmacies and private drugstores with average $2.00 \mathrm{DHD}$ for the capitals. Multiple linear regression analysis showed that $76 \%$ of the variation was explained by population density $(\beta=0.310 p=0.045)$ and percentage of physicians $(\beta=0.507 p=0.016)$. The consumption of short half-life anxiolytics has been on the increase, mainly in the cities of greater population density and concentration of physicians.
\end{abstract}

Key words Anxiolytic benzodiazepines, Consumption, Brazilian state capitals, Sociodemographics
Resumo O objetivo do artigo é conhecer a distribuição e a frequência de consumo de ansiolíticos benzodiazepinicos, bem como avaliar a correlação entre consumo e características demográficas, epidemiológicas, econômicas e sociais. Estudo ecológico tendo como unidade amostral as 27 capitais brasileiras. A coleta de dados foi executada através do banco da Anvisa, para a dispensação do Alprazolam, Bromazepam, Clonazepam, Diazepam e Lorazepam, de 2010 a 2012, do Censo Demográfico 2010 (IBGE), Datasus e da pesquisa Demografia Médica. A análise estatística descritiva e a de regressão linear múltipla foram realizadas para análise dos dados. A região Norte possui as capitais com menores médias de consumo desses medicamentos e o Sudeste as mais elevadas. O consumo médio para a população de todas as capitais foi de 3,60 DHD. O Alprazolam é o mais dispensado pelas farmácias e drogarias particulares, com média de 2,00 DHD para as capitais. A análise de regressão linear múltipla demonstrou que $76 \%$ da variância do consumo foi explicada pela variação da densidade demográfica $(\beta=0,310 p=0,045)$ e percentual de médicos $(\beta=0,507 p=0,016)$. $O$ consumo de ansioliticos de meia vida curta vem crescendo ao longo dos anos, principalmente nas capitais de maior densidade demográfica e concentração de médicos.

Palavras-chave Ansiolíticos benzodiazepínicos, Consumo, Capitais brasileiras, Sociodemográfica 


\section{Introdução}

Os ansiolíticos benzodiazepínicos (BDZ) alcançaram grande popularidade entre os membros da classe médica e na população nas décadas de 1970 e 1980, uma vez que demonstraram tamanha eficácia no combate da ansiedade, insônia, agressividade e convulsões, dentre outras ações, com menos efeitos depressores sobre o SNC1. Essa menor influência na inibição dos centros respiratórios propiciou uma sensação de segurança e maior desinibição no momento de indicar ou fazer uso desses medicamentos. Estima-se que os $\mathrm{BDZ}$ estejam entre os fármacos mais prescritos nos países ocidentais. Além disso, existe ainda uma média em que cada clínico teria por volta de 50 pacientes dependentes de benzodiazepínicos, e que destes, $50 \%$ desejam descontinuar o uso e $30 \%$ acreditam que os médicos chegam inclusive a estimular o uso da medicação².

Aspectos sociais sabidamente são providos da capacidade de colocar alguns grupos populacionais em desvantagem com relação à oportunidade de serem e de se manterem sadios ${ }^{3}$. Muito embora a prevalência geral dos transtornos mentais pareça não sofrer grande interferência do sexo, o mesmo não pode ser dito dos transtornos menores ou mais comuns, tais como ansiedade e depressão ${ }^{4}$. Em estudo produzido em uma das capitais da região Sul do Brasil, o consumo de ansiolíticos $\mathrm{BDZ}$ apresentou o dobro da prevalência entre as mulheres, quando comparado aos homens ${ }^{1}$.

Em certo grau, análises que correlacionam significativamente o consumo de ansiolíticos BDZ com determinantes sociais são precedidos por estudos que identificaram geograficamente a influência de fatores demográficos na prevalência de diversas outras condições de saúde mental. Assim, a ocorrência de correlação entre admissão em urgências psiquiátricas e marcadores de pobreza, privação socioeconômica, fragmentação social, isolamento individual e proximidade de serviços de saúde justificou a utilização de tais variáveis na busca por correlação com consumo de BDZ na França ${ }^{5}$. De forma semelhante, outros fatores, tais como idade ${ }^{6,7}$, mortes violentas, índices de suicídio ${ }^{8}$ e desemprego ${ }^{5,6,9}$ têm demonstrado relevância na tentativa de explicar o uso abusivo desses medicamentos ansiolíticos em regiões distintas de outros países. Índices compostos regularmente também são utilizados para melhor representar aspectos sociais complexos e distintos. O marcador de pobreza material, por exemplo, constitui um fator em que o nível de desemprego, renda e ocupação de moradia auxiliam na sua construção.
Nesse sentido, este trabalho objetiva conhecer a frequência e a distribuição do consumo, nas farmácias e drogarias privadas, de ansiolíticos benzodiazepínicos nas capitais brasileiras e avaliar a sua relação com características demográficas, epidemiológicas, econômicas e, sobretudo, sociais.

\section{Método}

\section{Unidade de análise}

Trata-se de um estudo ecológico em que Brasília e demais 26 capitais estaduais brasileiras constituem as unidades de análise. Estas configuram o centro administrativo e financeiro dos estados, historicamente foco de atração populacional, lugar de relevância na formulação de políticas públicas e alocação de recursos. Dentro dessa perspectiva, o IBGE ${ }^{10}$ tem buscado agregar indicadores ao nível municipal com o intuito de captar a extrema variedade de realidades sociais - historicamente presente nesse país de dimensões continentais - e evidenciar o processo de mudanças.

\section{Variáveis e fontes}

Neste estudo, foram utilizados apenas dados secundários. As informações referentes às condições socioeconômicas e demográficas da população - razão de sexo, percentual de idosos, densidade demográfica, taxas de analfabetismo, IDH e taxa de desemprego - tiveram como fonte o Censo Demográfico 2010 do Instituto Brasileiro de Pesquisa e Estatística (IBGE). Já para os dados epidemiológicos, como o número de óbitos por suicídio por cem mil habitantes, para o ano de 2010, originaram-se no Departamento de Informática do SUS (DATASUS). Também foram utilizados dados da pesquisa Demografia Médica no Brasil ${ }^{11}$, mais especificamente a densidade de médicos por mil habitantes nas capitais para o ano de 2011.

As informações referentes à dispensação dos medicamentos ansiolíticos derivados de benzodiazepínicos (Alprazolam, Bromazepam, Clonazepam, Diazepam e Lorazepam), oriundos de drogarias e farmácias privadas, foram fornecidas pela coordenação do Sistema Nacional de Gerenciamento de Produtos Controlados (SNGPC) da Agência Nacional de Vigilância Sanitária (ANVISA), em miligramas ( $\mathrm{mg}$ ) mensais de cada princípio ativo para os anos de 2010, 2011 e 2012. 


\section{Análise estatística}

Esses dados foram transformados em dose diária definida (DDD) por mil habitantes por dia (DHD) para Brasília e demais 26 capitais brasileiras, por medicamento e total de ansiolíticos (soma), de acordo com o sistema ATC/DDD ${ }^{12}$, como recomendação da Organização Mundial da Saúde (OMS) para estudos de utilização de medicamentos. Ainda foi realizada a média dos consumos das capitais ponderada pelas populações (média das médias).

Através do software SPSS Statistics 17.1, foi confeccionada a matriz de correlação de Pearson. Foram consideradas aptas a entrar no modelo variáveis com $p$-valor até o limite de 0,20 para correlação com a variável dependente. No modelo da análise de regressão linear múltipla, permaneceram as variáveis com $p$-valor $<0,05$, com exceção das variáveis explicativas "percentual de idoso" e "taxa de analfabetismo" como ajuste para o modelo. Utilizou-se o coeficiente de determinação $\left(\mathrm{R}^{2}\right)$ como medida de ajuste para o modelo e o teste de Shapiro-Wilk para verificar a distribuição normal dos resíduos, com limite de $p=0,05$.

\section{Resultados}

Durante a análise descritiva, observou-se ampla tendência de razões de sexo inferiores a um, uma média de $9,33 \%$ para o percentual de idosos, densidade demográfica variando de 12,57 a mais de 7.700, valores médios para as taxas de analfabetismo e IDH respectivamente de 7,92\% e 0,776; além disso, das quinze piores taxas de desemprego, apenas uma não pertence às regiões Norte ou Nordeste. A razão de médicos por mil habitantes, dentre todas as capitais, foi de 3,77, e $9,76 \%$ foi o percentual de pessoas que se autodenominam sem religião. A média de óbitos por suicídio dentre as capitais foi de 4,88 para cada grupo de cem mil habitantes.

O consumo, em conjunto, dos cinco importantes ansiolíticos benzodiazepínicos variou de apenas 0,24 DHD (Manaus) até 7,29 (Belo Horizonte), possuindo 3,04 como média e 2,30 de desvio padrão. No Gráfico 1, observa-se com facilidade o menor consumo para a média dos três anos, em todas as capitais da região norte do Brasil.

Com relação à evolução do consumo por medicamento, o único fármaco que se comportou diferente dos demais foi o Diazepam. Enquanto outros fármacos desta classe demonstram elevação seguida na média ponderada do consumo nas capitais em todos os anos, o mais antigo - e talvez mais famoso - segue um caminho diametralmente oposto, decaindo em número de DHD ano após ano (Gráfico 2). Não houve alternância de posição entre os fármacos quanto ao consumo de DHD. O Alprazolam manteve-se no topo, seguido por Bromazepam, Clonazepam, Lorazepam e Diazepam. A média para os três anos foi de 2,00 DHD para o Alprazolam, de 0,74 para o Bromazepam, 0,43 do Clonazepam, 0,11 para o Diazepam e 0,32 para o Lorazepam. Dessa forma, a população das capitais consumiu em média, para os três anos, 3,60 DHD dos cinco ansiolíticos benzodiazepínicos, e apenas em duas vezes o Al-

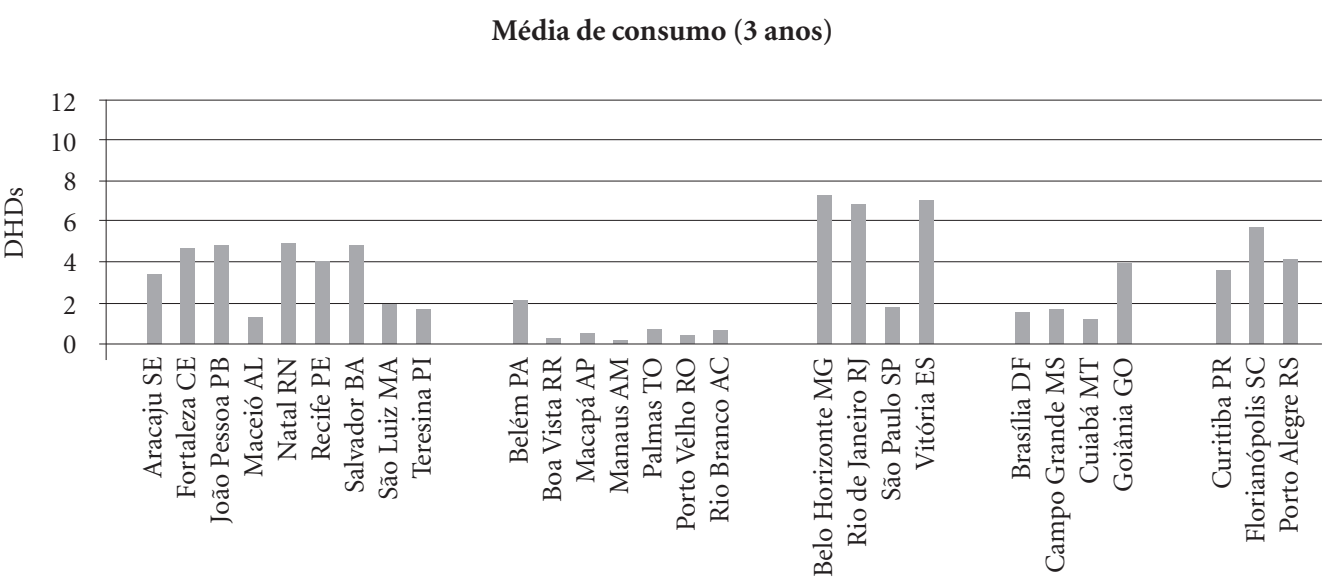

Gráfico 1. Distribuição média do consumo dos Ansiolíticos Benzodiazepínicos (Alprazolam, Bromazepam, Clonazepam, Diazepam e Lorazepam) entre 2010 e 2012 em número de DHD por capital. 
prazolam teve sua hegemonia maculada (em Belo Horizonte e São Luiz), e mesmo assim somente no ano de 2010. Quando observada a evolução do consumo ano a ano, e não mais a média dos três anos, tem-se o valor de 2,63 DHD em 2010, passando para 3,66 em 2011 e chegando a 4,53 em 2012. A diferença do consumo entre os anos de 2012 e 2010 representa um aumento de $72 \%$.

Quando observados os valores na matriz de correlações de Pearson e/ou na regressão linear simples, a taxa de desemprego e o percentual sem religião apresentaram $p$-valor superior a 0,2 ; os óbitos por suicídio, mesmo apresentando $p$-valor inferior no limite da significância estatística, não tiveram correlação com o consumo de ansiolíticos derivados de benzodiazepínicos (Tabela 1).

Ainda na análise de regressão linear simples, percebeu-se uma forte correlação positiva entre o percentual de idosos $(0,800)$ e a densidade de médicos $(0,791)$ com a média do consumo. Enquanto isso, a correlação da razão de sexo e da taxa de analfabetismo demonstrou sinal negativo, denotando redução do consumo de ansiolíticos com o aumento dessas variáveis explicativas.

Em modelo construído a partir da análise de regressão linear múltipla, foi possível explicar $76 \%$ da variância do consumo desses ansiolíticos. Nesse modelo, a densidade de médicos por habitantes, assim como a densidade demográfica das capitais, mantiveram significância estatística mesmo após o ajuste para o percentual de idosos e taxa de analfabetismo da população. Os coeficientes de regressão encontrados estão na Tabela 2.

A análise desse modelo revelou uma distribuição normal dos resíduos estandardizados com valor de significância superior a 0,05 , na estatística de Shapiro-Wilk e Kolmogorov-S- mirnov. Além disso, todos os pontos possuem distribuição aleatória no entorno do zero, caracterizando variância nula dos erros e também se apresentando dentro do intervalo de três desvios padrão positivo ou negativo, denotando ausência de valores discrepantes (outliers). São Paulo se destacou em tal análise ao ser a única capital a se aproximar desse limite, superando negativamente os dois desvios padrão.

\section{Discussão}

Embora os benzodiazepínicos sejam utilizados no tratamento de várias doenças psiquiátricas e não psiquiátricas e sejam geralmente seguros $\mathrm{e}$ bem tolerados, o potencial para a utilização indevida e abusiva desses depressores do SNC é considerável ${ }^{13}$. Trabalhos relacionados ao con-

Tabela 1. Coeficiente de Regressão Linear Simples entre variáveis independentes e o consumo de ansiolíticos benzodiazepínicos e valor de $p$.

\begin{tabular}{lcr}
\hline \multicolumn{1}{c}{ Variáveis } & p-valor & \multicolumn{1}{c}{$\beta$} \\
\hline Razão de Sexo & 0,000 & $-0,639$ \\
Percentual de Idosos & 0,000 & 0,800 \\
Densidade Demográfica & 0,000 & 0,661 \\
Taxa de Analfabetismo & 0,010 & $-0,490$ \\
IDH & 0,002 & 0,580 \\
Taxa de Desemprego & 0,352 & $-0,186$ \\
Percentual sem Religião & 0,641 & 0,094 \\
Renda per capita & 0,002 & 0,576 \\
Suicídios & 0,090 & $-0,333$ \\
Densidade de Médicos & 0,000 & 0,791 \\
\hline
\end{tabular}

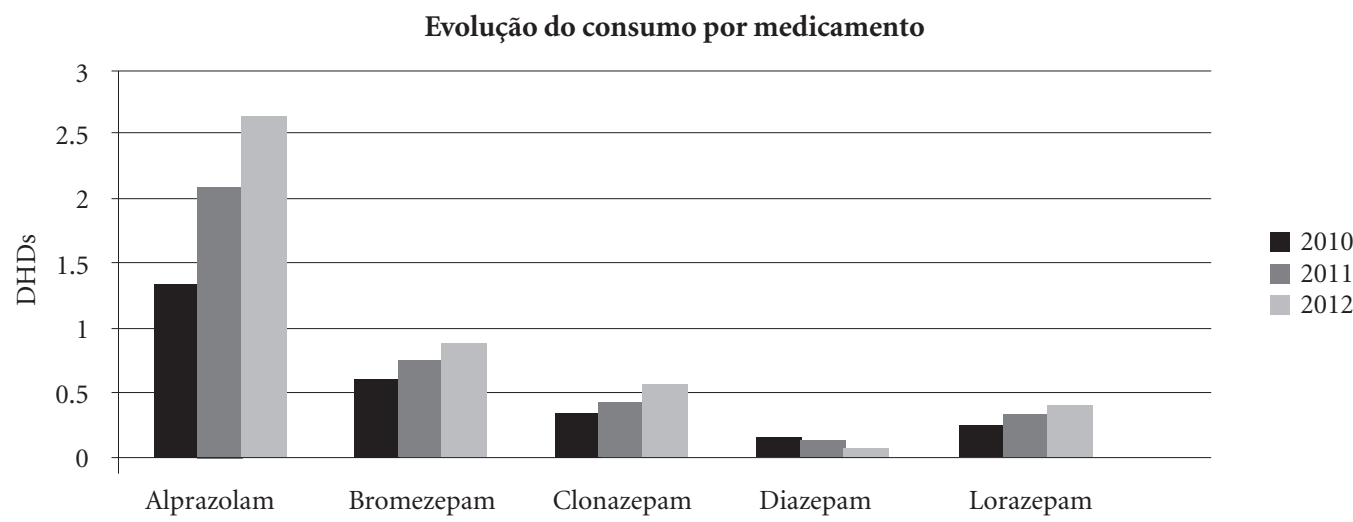

Gráfico 2. Evolução do Consumo anual por Benzodiazepínico em 2010, 2011 e 2012 em número de DHD. 
Tabela 2. Coeficientes do Modelo de Regressão Linear Múltipla.

\begin{tabular}{|c|c|c|c|c|c|}
\hline \multirow[b]{2}{*}{ Modelo } & \multicolumn{5}{|c|}{ Coeficientes $^{a}$} \\
\hline & $\beta$ & $\begin{array}{c}\text { Erro } \\
\text { Padrão }\end{array}$ & $\begin{array}{c}\text { Coeficientes } \\
\text { estandardizados } \\
\beta\end{array}$ & t & Sig. \\
\hline \multicolumn{6}{|l|}{1} \\
\hline (Constante) & 0.268 & 1.331 & & 0.202 & 0.842 \\
\hline Percentual de Idosos & 0.085 & 0.186 & 0.108 & 0.456 & 0.653 \\
\hline Densidade Demográfica & $2.66 \times 10^{-4}$ & $1.17 \times 10^{-4}$ & 0.310 & 2.123 & 0.045 \\
\hline Taxa de Analfabetismo & -0.069 & 0.059 & -0.138 & -1.174 & 0.253 \\
\hline Densidade Médicos & 0.493 & 0.189 & 0.507 & 2.611 & 0.016 \\
\hline
\end{tabular}

${ }^{a}$ Dependente variável: média 3 anos.

sumo desses medicamentos são frequentes em países desenvolvidos, mas escassos em países em desenvolvimento ${ }^{1}$. Parte dessa escassez deve-se à dificuldade, pelo menos relativa, em conseguir informações referentes ao consumo, mesmo de medicamentos controlados. Até bem pouco tempo, o livro de controlados constituía a única forma de acesso a dados de forma relativamente rápida para o desenvolvimento de um estudo local, mas simplesmente impensável no âmbito nacional ou até mesmo regional.

A partir da consolidação do SNGPC - para coleta, processamento, análise e transmissão das informações da dispensação dos produtos controlados -, obteve-se uma maior agilidade na disponibilização de alguns dados relativos à prescrição e aquisição como sinônimos de consumo. A necessidade de aprimoramento deve ser contínua, e fica patente na ausência de informações para algumas capitais, fato que levou à exclusão tanto do ano de 2009, quanto da dispensação de medicamentos manipulados do presente estudo. Quando comparado ao sistema utilizado por trabalhadores franceses ${ }^{5}$ - em que um cartão com chip registra e envia eletronicamente os dados da compra e do usuário para o Seguro de Saúde Geral, impedindo inclusive a aquisição de quantidades não justificadas -, vislumbra-se o quanto ainda é possível avançar.

Estudos anteriores têm verificado a prevalência de uso de benzodiazepínicos sem receita médica variando entre $3,3 \%{ }^{14}$ e $8,4 \%{ }^{1}$. Tal achado é corroborado com a identificação do descuido no preenchimento das notificações de receita ou até com indícios de falsificação, como numeração repetida e número do Conselho de médico já falecido ${ }^{14}$. Uma medida mais imediata talvez seja a interação entre os softwares de controle de venda/estoque das farmácias e drogarias e o de transmissão (SNGPC).

Torna-se relevante insistir na necessidade de aprimoramento do mecanismo de controle, tendo em vista a correlação existente entre a aquisição sem receita e o uso indevido. Um estudo realizado entre jovens universitários americanos encontrou forte associação entre o consumo de benzodiazepínicos não prescritos com comportamentos de risco, como bebedeira, tabagismo, uso de opioides, cocaína, estimulantes, bem como dirigir ou pegar carona com alcoolizados ${ }^{15}$.

Passando a avaliar apenas os dados de consumo informado, sem levar em conta a adequação do uso, chega-se ao total de 3,60 DHD para os cinco ansiolíticos derivados de benzodiazepínicos estudados. Ou seja, para cada grupo de dez mil moradores das capitais brasileiras, em média 36 fizeram uso de uma dose durante todos os dias do ano. Comparativamente a outros países ${ }^{9,16-19}$, onde estudos nesse sentido são realizados há mais tempo, a média das capitais brasileiras pode ser considerada baixa. Na Sérvia, por exemplo, chegou a ocorrer um ápice de 133 DHD em período pós-bombardeio da Organização do Tratado do Atlântico Norte (OTAN), mas habitualmente oscila entre 3,7 e 20,4 DHD.

Esses dados se tornam um pouco mais preocupantes quando associados à informação de que na Itália, por exemplo, mais da metade dos usuários crônicos de BDZ costuma utilizar dose inferior à meia $\mathrm{DDD}^{9}$. Se isso por um lado é positivo, por se reconhecer que usualmente não ocorre superdosagem, tem-se também a percepção de que o universo de usuários é subestimado pelo valor da DHD. O contexto negativo advém da premissa de que não há comprometimento em usar a dose idealmente definida de ansiolíticos, o mal está em 
fazer uso prolongado em uma parcela maior da população, mesmo em subdoses.

Igualmente chama a atenção a possibilidade de aumento recorrente na taxa de consumo desses fármacos. As capitais brasileiras passaram de um consumo de 2,63 DHD, em 2010, para 3,66 em 2011, chegando a 4,53 em 2012. Em termos absolutos, foi acrescentado mais um usuário em cada grupo de mil moradores em cada ano que passou. Não parece ser muito quando comparado ao crescimento espanhol de 2,78 DHD ao ano no período entre 1995 e $2002^{16}$; contudo, no plano relativo, representa um aumento de mais de $70 \%$ em um intervalo de dois anos.

$\mathrm{Na}$ análise individual dos medicamentos, existe um revezamento dentre os mais populares da classe, dependendo tanto do local do estudo como da época. Em comum, existe uma tendência mais atual de optar por medicamentos com meia vida curta, tais como: Alprazolam, Bromazepam e Lorazepam ${ }^{2}$. Os médicos brasileiros parecem estar seguindo tal tendência, uma vez que Alprazolam e Bromazepam figuram como os mais prescritos e apresentaram aumento na dispensação, quando comparado aos anos anteriores. $\mathrm{O}$ Diazepam contraria resultados que apontavam para elevado e crescente consumo ${ }^{19}$, e demonstra seguir a tendência de, como benzodiazepínico de meia vida intermediária, ser preterido ${ }^{17}$, provavelmente em benefício dos de meia vida mais curta. Diferente ocorre com o Clonazepam que, mesmo possuindo meia vida intermediária, foi o terceiro mais consumido e de forma crescente. Talvez seja possível atribuir esse resultado à sua ação anticonvulsivante, fator que leva a OMS a classificá-lo como anticonvulsivante derivado de benzodiazepínico, e não como ansiolítico.

Existe dúvida ainda de como essa tendência de redução de medicamentos com maior meia vida vem ocorrendo, tendo em vista a inexistência de qualquer intervenção educacional maior para os prescritores generalistas. Em estudo desenvolvido no Canadá, levantou-se a possibilidade tanto da difusão passiva da informação para os médicos quanto do aumento da disponibilidade de terapias comportamentais ${ }^{20}$.

Diferentemente dos resultados descritivos, a análise dos fatores intervenientes no consumo de ansiolíticos apresentou bastante controvérsia, a começar pelo desemprego que em estudos realizados tanto com a população italiana ${ }^{9}$, como dos Países Baixos ${ }^{6}$, em que encontraram relação significante e positiva, mesmo em análise múltipla, enquanto que para a população das capitais brasileiras nem mesmo uma correlação simples existia. Fato semelhante ocorreu para suicídios, não confirmando a influência demonstrada em outro estudo ${ }^{8}$.

Outra característica mais claramente relacionada aos grandes centros urbanos brasileiros demonstra relevância na composição da variável resposta. A densidade demográfica figura como um dos pilares atuais da saúde urbana ${ }^{21}$, possivelmente personificando um conjunto de variáveis de difícil mensuração se não diretamente relacionada à qualidade de vida, certamente determinante para o bem-estar dos habitantes. Os atributos físicos e sociais (contexto) da cidade demonstram potencial para afetar a saúde e muitas vezes encontram-se espacialmente associados $^{22}$. Trânsito caótico, sensação de insegurança ${ }^{23}$, ambiente competitivo ${ }^{24}$, grande apelo consumista ${ }^{25}$ e baixa coesão social ${ }^{26}$ são algumas das características que compõem o estilo de vida nas grandes cidades atuais, fato que se repete nos grandes adensamentos populacionais do Brasil. O desafio de sobreviver saudável a essa realidade parece impraticável para uma parcela nada desprezível da sociedade.

Associado a essa realidade, tem-se a crença reforçada através de prescrições pouco criteriosas - de que os ansiolíticos benzodiazepínicos desempenhariam o papel de um tônico para saúde mental na ausência de tratamentos mais efetivos, sobretudo para populações mais carentes ${ }^{27}$. Esse grupo de medicamento tem se demonstrado útil como sintomático para patologias psiquiátricas e não psiquiátricas. $\mathrm{O}$ que precisa ficar claro é a existência de alternativas igualmente eficazes para o controle de sintomatologia ansiosa e que essas não passam necessariamente por terapia medicamentosa.

Tal posicionamento deve firmar-se como alternativa também ao modelo biomédico, que exerce influência definitiva sobre os currículos das escolas médicas. Apesar do sucesso logrado pela medicina com a incorporação da pesquisa experimental, biologia e pela especialização, sua associação à indústria farmacêutica produziu alguns efeitos adversos ${ }^{28}$. A incorporação pelo médico da ideia do fármaco como o principal caminho - se não o único - para responder às demandas que lhe chegam constitui uma política vantajosa para a indústria e preocupante para a saúde da população ${ }^{29}$.

No Reino Unido, existe a recomendação de terapia não farmacológica inicial e, em ocorrendo insucesso, a introdução de benzodiazepínicos de curta duração, ou Zopiclone. A terapia cognitivo comportamental, os inibidores seletivos da recaptação da serotonina e mesmo os antidepressivos tricíclicos são preferíveis aos ansiolíticos benzo- 
diazepínicos para o tratamento da ansiedade ${ }^{7}$. Mesmo nos casos em que a introdução dos BDZ revela-se necessária, os guidelines orientam a fazê-lo por períodos de até três meses. O que parece óbvio, contudo, é a predisposição, por parte de quem prescreve, em evitar confronto com os usuários crônicos, bem como o desconhecimento sobre o potencial de dependência dos psicotrópicos. Em resultado de avaliação, ficou evidente essa imprecisão por parte de médicos postulantes à residência, da insignificância do potencial de dependência dos antidepressivos quando comparado aos ansiolíticos ${ }^{1}$, justificando o uso crônico indevido mesmo sob supervisão médica ${ }^{30}$.

Uma vez sendo o acesso ao médico fator decisivo para o consumo, como parecia lógico e ficou evidenciado com o coeficiente de regressão significante e positivo, investir na disseminação desses conhecimentos dentre esses profissionais parece igualmente racional como medida de desestímulo ao consumo. Outras medidas - como o acompanhamento multiprofissional (médico, enfermeiro, farmacêutico e auxiliar de enfermagem) - demonstraram potencial para reduzir em mais de um terço o consumo desses fármacos, mesmo diante de sua clientela mais fiel, ou seja, os idosos institucionalizados ${ }^{31}$. A maior fiscalização e a limitação ao uso puramente, demonstra potencial ainda superior na redução do consumo, muito embora tenha provocado, no início da década de 1990, na população de Nova York, uma escalada na dispensação de fármacos menos apropriados $^{32}$.

O modelo explicativo adotado no presente estudo resultou do teste de diversas outras variáveis, a fim de explicar significativa parcela da variância do consumo de ansiolíticos. A ausência de determinantes no modelo escolhido - não unânimes, mas recorrentes em outros estudos, tal como o gênero, representado pela razão de sexo - pode ter motivação estatística. A amostra de quantidade limitada permite que apenas poucos previsores, somente com os maiores efeitos, demonstrem significância no modelo ${ }^{33}$.

Constitui limitação, também, sobretudo para efeito de comparação com o consumo em outras regiões, a ausência dos demais membros da classe dos ansiolíticos benzodiazepínicos e mesmo não benzodiazepínicos. Outra possível fonte de viés consiste na utilização de informações apenas oriundas de farmácias e drogarias particulares, muito embora se trate de medicamentos reconhecidamente baratos ${ }^{14}$.

Esses medicamentos largamente utilizados, marcadamente no Ocidente, representaram um grande avanço no tratamento de diversas doenças, psiquiátricas ou não. Atualmente, seu consumo também pode ser interpretado como problemático, tendo em vista a frequência de mau uso ou o consumo indiscriminado. É importante que fique claro que as pessoas terão que passar por acontecimentos tanto positivos e desejáveis quanto indesejáveis, durante a vida, e as duas possibilidades resultarão em certa carga de estresse psicológico com consequências muito variáveis. Esses eventos seriam componentes das vivências do indivíduo e irão interagir com fatores genéticos, de personalidade, aptidão e também com as condições e estilo de vida individuais ${ }^{4}$.

Uma questão que se destaca é a atual intolerância ao sofrimento. O ideal de perfeição contemporâneo muitas vezes é conquistado através do consumo, até mesmo de medicamentos ${ }^{34}$. Vale reiterar que os ansiolíticos são úteis, seguros, e devem ser empregados quando bem indicados.

Diante do exposto, pode-se concluir que o aumento do consumo dos benzodiazepínicos nas capitais de maior densidade demográfica e percentual de médicos se deve à crescente medicalização da sociedade moderna, refletida na formação dos profissionais médicos.

Parece claro ainda que, no caminho mais curto para se chegar ao denominador de consumo otimizado, estariam a valorização da educação médica continuada e o estímulo às parcerias multiprofissionais. A partir do atual estudo, também seria fundamental o direcionamento dos investimentos públicos no sentido de assegurar boas condições de vida nas pequenas e médias cidades, na tentativa de desestimular o crescimento das grandes cidades e enormes densidades demográficas.

\section{Colaboradores}

AA Araújo e MAF Ferreira participaram da elaboração e revisão do artigo. AJP Azevedo participou de todas as etapas de construção do artigo. 


\section{Referências}

1. Kapczinski F, Amaral OB, Madruga M, Quevedo J, Busnello JV, de Lima MS. Use and misuse of benzodiazepines in brazil: a review. Subst Use Misuse 2001; 36(8):1053-1069.

2. Nastasy H, Ribeiro M, Marques ACPR. Abuso e Dependência dos Benzodiazepínicos. Rio de Janeiro: Associação Brasileira de Psiquiatria; 2008.

3. Barata RB. Como e por que as desigualdades sociais fazem mal à saúde. Rio de Janeiro: Fiocruz; 2009.

4. Organização Mundial da Saúde (OMS). Relatório mundial da saúde: Saúde mental: nova concepção, nova esperança. Genebra: OMS; 2002.

5. Bocquier A, Bezzou K, Nauleau S, Verger P. Dispensing of anxiolytics and hypnotics in southeastern France: demographic factors and determinants of geographic variations. Fundam Clin Pharmacol 2008; 22(3):323-333.

6. Manthey L, van Veen T, Giltay EJ, Stoop JE, Neven AK, Penninx BW, Zitman FG. Correlates of (inappropriate) benzodiazepine use: the Netherlands Study of Depression and Anxiety (NESDA). Br J Clin Pharmacol 2011; 71(2):263-272.

7. Hollingworth SA, Siskind DJ. Anxiolytic, hypnotic and sedative medication use in Australia. Pharmacoepidemiol Drug Saf 2010; 19(3):280-288.

8. Sundquist J, Ekedahl A, Johansson SE. Sales of tranquillizers, hypnotics/sedatives and antidepressants and their relationship with underprivileged area score and mortality and suicide rates. Eur J Clin Pharmacol 1996; 51(2):105-109.

9. Magrini N, Vaccheri A, Parma E, D’Alessandro R, Bottoni A, Occhionero M, Montanaro N. Use of benzodiazepines in the Italian general population: prevalence, pattern of use and risk factors for use. Eur J Clin Pharmacol 1996; 50(1-2):19-25.

10. Instituto Brasileiro de Geografia e Estatística (IBGE). Indicadores Sociais Municipais: Uma Análise dos Resultados do Universo do Censo Demográfico 2010. Rio de Janeiro: IBGE; 2011.

11. Scheffer M, Biancarelli A, Cassenote A. Demografia Médica no Brasil: dados gerais e descrições de desigualdades. São Paulo: Conselho Regional de Medicina do Estado de São Paulo, Conselho Federal de Medicina; 2011.

12. WHO Collaborating Centre for Drug Statistics Methodology. Guidelines for ATC classification and DDD assignment 2010. Oslo: WHO; 2009.

13. Mota DM, Costa AA, Teixeira CS, Bastos AA, Dias MF. Uso abusivo de benzidamina no Brasil: uma abordagem em farmacovigilância. Cien Saude Colet 2010; 15(3):717-724.

14. Orlandi P, Noto AR. Uso indevido de benzodiazepínicos: um estudo com informantes-chave no município de São Paulo. Rev. Latino-Am. Enfermagem 2005; 13 (n. esp):896-902.

15. McCabe SE. Correlates of nonmedical use of prescription benzodiazepine anxiolytics: results from a national survey of U.S. college students. Drug Alcohol Depend 2005; 79(1):53-62.

16. Garcia del Pozo J, Iglesias FJA, García-Pando AC, Corominas DM, Sanz MM, Pozo VG. Utilización de ansiolíticos e hipnóticos en España (1995-2002). Rev. Esp. Salud Publica 2004; 78(3):379-387.

17. Bernik MA, Soares MBM, Soares CN. Benzodiazepínicos padrões de uso, tolerância e dependência. Arq. Neuro-Psiquiatr. 1990; 48(1):131-137.
18. Portugal. Ministério da Saúde. Evolução do consumo de benzodiazepinas em Portugal de 1995 a 2001. Lisboa: Observatório do Medicamento e dos Produtos de Saúde; 2002.

19. Firmino KF, Abreu MHNG, Perini E, Magalhães SMS. Utilização de benzodiazepínicos no Serviço Municipal de Saúde de Coronel Fabriciano, Minas Gerais. Cien Saude Colet 2012; 17(1):157-166.

20. Tu K, Mamdani MM, Hux JE, Tu JB. Progressive trends in the prevalence of benzodiazepine prescribing in older people in Ontario, Canada. J Am Geriatr Soc 2001; 49(10):1341-1345.

21. Caiaffa WT, Ferreira FR, Ferreira AD, Oliveira CDL, Camargos VP, Proietti FA. Saúde urbana: "a cidade é uma estranha senhora, que hoje sorri e amanhã te devora". Cien Saude Colet 2008; 13(6):1785-1796.

22. Clougherty JE, Kubzansky LD. A framework for examining social stress and susceptibility to air pollution in respiratory health. Cien Saude Colet 2010; 15(4):20592074.

23. Souza ER, Lima MLC. Panorama da violência urbana no Brasil e suas capitais. Cien Saude Colet 2006; 11(Supl.):1211-1222.

24. Sweeting H, West P, Young R, Der G. Can we explain increases in young people's psychological distress over time? Soc Sci Med 2010; 71(10):1819-1830.

25. Eckersley R. Is modern Western culture a health hazard?: Point-counterpoint. Int J Epidemiol 2006; 35(2):252-258.

26. Johnell K, Lindström M, Melander A, Sundquist J, Eriksson C, Merlo J. Anxiolytic-hypnotic drug use associated with trust, social participation, and the miniaturization of community. Soc Sci Med 2006; 62(5):1205-1214.

27. Quigley P, Usher C, Bennett K, Feely J. Socioeconomic Influences on Benzodiazepine Consumption in an Irish Region. Eur Addict Res 2006; 12(3):145-150.

28. Carvalho YM, Ceccim RB. Formação e educação em saúde: aprendizados com a saúde coletiva. In: Campos GWS, Minayo MCS, Akerman M, Drumond Júnior M, Carvalho YM. Tratado de saúde coletiva. Rio de Janeiro: Hucitec, Fiocruz; 2006. p. 149-182.

29. Tesser CD. Medicalização social (II): limites biomédicos e propostas para a clínica na atenção básica. Interface (Botucatu) 2006; 10(20):347-362.

30. Souza ARL, Opaleye ES, Noto AR. Contextos e padrões do uso indevido de benzodiazepínicos entre mulheres. Cien Saude Colet 2013; 18(4):1131-1140.

31. Schmidt I, Claesson CB, Westerholm B, Nilsson LG, Svarstad BL. The impact of regular multidisciplinary team interventions on psychotropic prescribing in Swedish nursing homes. J Am Geriatr Soc 1998; 46(1):77-82.

32. McNutt LA, Coles FB, McAuliffe T, Baird S, Morse DL, Strogatz DS, Baron RC, Eadie JL. Impact of regulation on benzodiazepine prescribing to a low income elderly population, New York State. J Clin Epidemiol 1994; 47(6):613-25.

33. Field A. Regressão. In: Field A. Descobrindo a estatística usando o SPSS. 2a ed. Porto Alegre: Artmed; 2009. p. 156-220.

34. Amaral JGPD. Os destinos da tristeza na contemporaneidade [dissertação]. Rio de Janeiro: Pontifícia Universidade Católica; 2006.

Artigo apresentado em 03/10/2014

Aprovado em 15/05/2015

Versão final apresentada em 17/05/2015 